\title{
Compressed Sensing SAR Imaging for Wideband Linear Frequency Modulated Signal
}

\author{
Feifei Zhang ${ }^{a}$, Yaoliang Song ${ }^{b}$ and Tong $\mathrm{Mu}^{\mathrm{c}}$
}

School of Electronic Engineering and Optoelectronic Technology, Nanjing University of Science and Technology, Nanjing 210094, China.

a feifei_zhang1992@163.com, bylsong@mail.njust.edu.cn, c tonymu93@gmail.com

Keywords: compressed sensing, SAR, linear frequency modulated signal, sparse matrix, stretch processing.

\begin{abstract}
Compressed sensing (CS) theory provides a new chance to reduce the data rate of high resolution radar imaging system. A novel SAR imaging method based on compressed sensing for wideband linear frequency modulated (LFM) signal is proposed in this paper. The radar data compression is conducted in range and azimuth dimension respectively. A new sparse matrix based on stretch processing and Fourier transform is constructed and then applied to obtain the range profile information. Based on the range compression, the phase compensation and the azimuth compression are conducted. The method incorporates coherent mixing processing in sparse matrix, consequently simplifies hardware design of radar system. Simulation results show the effectiveness of the method in reducing data rate and suppressing sidelobe compared with conventional stretch processing (SP) method. It also turns out that the proposed method is robust in the case of serious noise.
\end{abstract}

\section{Introduction}

Synthetic aperture radar (SAR) imaging technology can produce the images of the stationary surface targets and terrain in all weather conditions. Wideband linear frequency modulated (LFM) signal is widely used in SAR system for high imaging resolution. With the imaging area getting larger and imaging resolution getting higher, more and more data will be gained in SAR system which leads to the burden of the system. The compressed sensing (CS) theory proposed in recent years breaks through the constraints of the conventional Nyquist sampling theorem [1-2]. This theory indicates that a sparse or compressible signal can be reconstructed from a small set of measurement values by a specifically designed nonlinear reconstruct algorithm.

In the past few years, many applications based on CS have been explored and researched in radar imaging area [3-5]. In the framework of CS theory, taking full advantage of sparsity or compressibility of echo signal, it is sufficient to use only a small number of samples to reconstruct high resolution images of targets. A compressed radar imaging method is proposed in [6]. It has two significant improvements: removing the requirement for the matched filter and reducing the bandwidth of the ADC. A novel strategy for SAR imaging based on CS is provided in [7]. It proposes that SAR image formation can be separated into two 1-D processing operations: range and azimuth compression. Then the CS processing can be applied to each dimension respectively. Some effective SAR compress processing methods in two dimensions are proposed in [8-11]. These methods above are mainly fit for baseband signal gained from coherent mixing processing. Besides, these methods need to predefine the observation target space and construct the sparse matrix by synthetizing the radar model data for each discrete spatial position. In [12], the stretch processing of LFM echoes is reformulated in matrix form and an orthogonal dictionary is established, but the specific implementation in radar imaging is not discussed.

A novel SAR imaging method based on CS for wideband LFM signal is proposed in this paper. In the method, the CS processing is applied to range and azimuth dimension respectively. Firstly, a sparse matrix based on stretch processing and Fourier transform in range is constructed to realize the sparsity of the radar data. Then, the range profile information is reconstructed from random measurements by orthogonal matching pursuit (OMP) algorithm. Based on the range compression, 
the phase compensation and stretch processing in azimuth are conducted. Finally, the azimuth compression based on Fourier transform matrix is conducted to obtain targets images. The method incorporates coherent mixing processing in sparse matrix, consequently simplifies the hardware design in radar system. Simulations demonstrate the effectiveness of the method in the data rate reduction and the sidelobe suppression.

This paper is organized as follows. In Section 2, the SAR imaging based on stretch processing is introduced. In Section 3, the SAR imaging method based on CS is discussed in detail. In Section 4, simulation results are presented to prove the validity of the proposed method and Section 5 concludes the paper.

\section{SAR Imaging Based on Stretch Processing}

Stretch processing (SP) used in wideband LFM signal, can effectively reduce the instantaneous bandwidth. The echo signal is stretched by multiplying it with the reference LFM signal that has the same sweep rate as the transmitted signal. Then the output signal spectrum is analyzed to detect the targets. SAR imaging geometry is shown in Fig.1, $H$ is SAR platform height, $\mathbf{P}$ is the radar position vector with uniform motion of $v, \mathbf{T}$ is the target position vector and $r\left(\eta, r_{0}\right)=\sqrt{r_{0}^{2}+(v \eta)^{2}}$ is instantaneous slant range where $r_{0}$ denotes the slant range of closest approach.

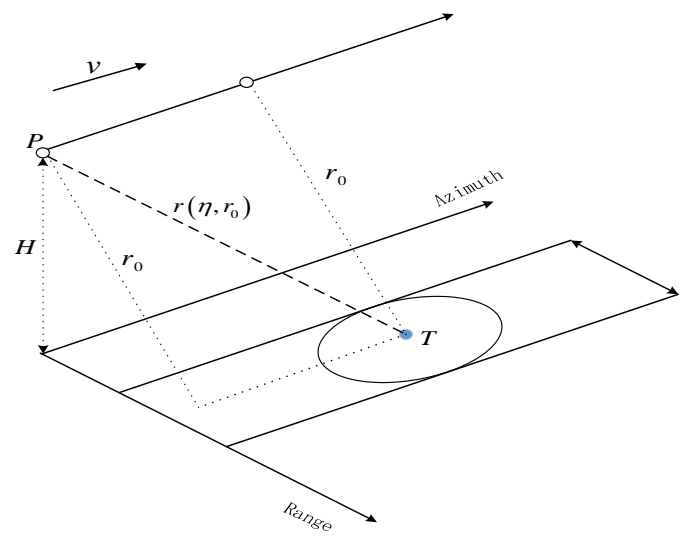

Fig. 1 SAR imaging geometry

Assume that the transmitted signal is based on LFM waveform, the echo signal from target is

$$
s_{r}(t ; \eta)=\sigma \cdot r e c t\left(\frac{t-2 r\left(\eta, r_{0}\right) / c}{T_{r}}\right) \exp \left[j 2 \pi f_{0}\left(t-\frac{2 r\left(\eta, r_{0}\right)}{c}\right)+j \pi K_{r}\left(t-\frac{2 r\left(\eta, r_{0}\right)}{c}\right)^{2}\right]
$$

where $T_{r}$ is the pulse duration in range, $f_{0}$ is the carrier frequency, $K_{r}=B / T_{r}$ is the range chirp rate, $\sigma$ is the target reflection coefficient, $t$ is the fast time and $\eta$ is the slow time.

Suppose the reference signal is

$$
s_{\text {ref }}(t)=\operatorname{rect}\left(\frac{t-2 r_{\text {ref }} / c}{T_{\text {ref }}}\right) \exp \left[j 2 \pi f_{0}\left(t-\frac{2 r_{\text {ref }}}{c}\right)+j \pi K_{r}\left(t-\frac{2 r_{\text {ref }}}{c}\right)^{2}\right]
$$

where $r_{\text {ref }}$ is the reference distance and $T_{\text {ref }}$ is the pulse duration of the reference signal.

After stretch processing, it yields

$$
s_{i f}(t ; \eta)=s_{r}(t ; \eta) \cdot s_{r e f}{ }^{*}(t)=\sigma \cdot r e c t\left(\frac{t-2 r_{\Delta} / c}{T_{r}}\right) \exp \left[-j \frac{4 \pi}{c} K_{r}\left(t-\frac{2 r_{r e f}}{c}\right) r_{\Delta}\right] \exp \left(-j \frac{4 \pi}{c} f_{0} r_{\Delta}\right) \exp \left[j 4 \pi K_{r}\left(\frac{r_{\Delta}}{c}\right)^{2}\right]
$$

where $r_{\Delta}=r\left(\eta, r_{0}\right)-r_{\text {ref }}$, taking Fourier transform of Eq.(3) in terms of fast time $t$, the range profile can be obtained

$$
S_{i f}\left(f_{r} ; \eta\right)=\sigma \cdot T_{r} \sin c\left[T_{r}\left(f_{r}+\frac{2 K_{r}}{c} r_{\Delta}\right)\right] \exp \left(-j \frac{4 \pi}{c} f_{0} r_{\Delta}\right) \exp \left[j 4 \pi K_{r}\left(\frac{r_{\Delta}}{c}\right)^{2}\right]
$$

After compensating residual video phase (RVP) and neglecting range migration correction, conduct stretch processing in azimuth, it can be expressed as 


$$
S\left(f_{r} ; \eta\right)=\sigma \cdot T_{r} r e c t\left(\frac{\eta}{T_{a}}\right) \sin c\left\{T_{r}\left[f_{r}+\frac{2 K_{r}}{c}\left(r_{0}-r_{\text {ref }}\right)\right]\right\} \exp \left(-j \frac{4 \pi}{c} f_{0} r_{0}+j \pi K_{a} \eta_{0}^{2}\right) \exp \left(j 2 \pi K_{a} \eta \eta_{0}\right)
$$

where $T_{a}, \eta_{0}$ and $K_{a}$ denote the pulse duration in azimuth, the time delay of the reference signal in azimuth and the azimuth chirp rate, respectively.

Taking Fourier transform of Eq.(5) in terms of slow time $\eta$, the target image can be obtained

$$
S\left(f_{r} ; f_{a}\right)=\sigma \cdot T_{r} T_{a} \sin c\left\{T_{r}\left[f_{r}+\frac{2 K_{r}}{c}\left(r_{0}-r_{r e f}\right)\right]\right\} \sin c\left[T_{a}\left(f_{a}-K_{a} \eta_{0}\right)\right] \exp \left(-j \frac{4 \pi}{c} f_{0} r_{0}+j \pi K_{a} \eta_{0}^{2}\right)
$$

The peaks of $\left|S\left(f_{r} ; f_{a}\right)\right|$ appear at $f_{r}=-\frac{2 K_{r}}{c}\left(r_{0}-r_{r e f}\right)$ and $f_{a}=K_{a} \eta_{0}$, corresponding to range and azimuth profile, respectively.

\section{SAR Imaging Method Based on CS}

\subsection{Principle of Compressed Sensing}

Suppose a discrete-time signal $\mathbf{x} \in \mathbb{C}^{N}$ is $K$ sparse or compressible on a set of basis and it can be expressed as

$$
\mathbf{x}=\sum_{i=1}^{N} \psi_{i} \alpha_{i}=\boldsymbol{\Psi} \boldsymbol{\alpha}
$$

where $\boldsymbol{\Psi}=\left[\psi_{1}, \psi_{2}, \cdots, \psi_{N}\right] \in \mathbb{C}^{N \times N}$ is the sparse basis matrix and $\boldsymbol{\alpha}$ is an $N \times 1$ vector with $K$ non-zero elements. The signal $\mathbf{x}$ is sparse or compressible in $\boldsymbol{\Psi}$ domain with $K$ sparsity.

When a measurement matrix $\boldsymbol{\Phi}$ is designed, we can get low-dimensional measurements $\mathbf{y}$ from the high-dimensional signal $\mathbf{x}$ through the nonlinear projection

$$
\mathbf{y}=\boldsymbol{\Phi x}=\boldsymbol{\Phi} \boldsymbol{\Psi} \alpha
$$

where $\boldsymbol{\Phi}$ is an $M \times N(M \ll N)$ measurement matrix and $\mathbf{y}$ is an $M \times 1$ vector.

The reconstruct of the signal $\mathbf{x}$ from the measurements $\mathbf{y}$ is ill-posed because of $M<N$. However, if $\mathbf{\Phi} \boldsymbol{\Psi}$ satisfies the restricted isometry property (RIP), the sparse representation of $\mathbf{x}$ can be exactly reconstructed by solving an $l_{1}$ norm problem

$$
\min \|\boldsymbol{\alpha}\|_{1} \quad \text { s.t. } \quad \mathbf{y}=\boldsymbol{\Phi} \Psi \boldsymbol{\alpha}
$$

This optimization problem can be solved by conventional linear programming techniques. The orthogonal matching pursuit (OMP) is an iterative greedy algorithm which can reconstruct the signal $\mathbf{x}$ from the measurements $\mathbf{y}$.

\subsection{SAR Imaging Method Based on CS}

Firstly, the sparse matrix based on stretch processing and Fourier transform in range and the sparse matrix based on Fourier transform in azimuth are constructed respectively. The $s_{r}(t ; \eta), s_{\text {ref }}(t), s_{i f}(t ; \eta)$ and $S\left(f_{r} ; \eta\right)$ can be discretized as

$$
\begin{aligned}
& s_{r}\left(t_{n} ; \eta_{l}\right)=\sigma \cdot r e c t\left(\frac{t_{n}-2 r\left(\eta_{l}, r_{0}\right) / c}{T_{r}}\right) \exp \left[j 2 \pi f_{0}\left(t_{n}-\frac{2 r\left(\eta_{l}, r_{0}\right)}{c}\right)+j \pi K_{r}\left(t_{n}-\frac{2 r\left(\eta_{l}, r_{0}\right)}{c}\right)^{2}\right] \\
& s_{\text {ref }}\left(t_{n}\right)=\operatorname{rect}\left(\frac{t_{n}-2 r_{r e f} / c}{T_{\text {ref }}}\right) \exp \left[j 2 \pi f_{0}\left(t_{n}-\frac{2 r_{r e f}}{c}\right)+j \pi K_{r}\left(t_{n}-\frac{2 r_{r e f}}{c}\right)^{2}\right] \\
& s_{i f}\left(t_{n} ; \eta_{l}\right)=\sigma \cdot r e c t\left(\frac{t_{n}-2 r_{\Delta} / c}{T_{r}}\right) \exp \left[-j \frac{4 \pi}{c} K_{r}\left(t_{n}-\frac{2 r_{r e f}}{c}\right) r_{\Delta}\right] \exp \left(-j \frac{4 \pi}{c} f_{0} r_{\Delta}\right) \exp \left[j 4 \pi K_{r}\left(\frac{r_{\Delta}}{c}\right)^{2}\right] \\
& S\left(f_{r} ; \eta_{l}\right)=\sigma \cdot T_{r} r e c t\left(\frac{\eta_{l}}{T_{a}}\right) \sin c\left\{T_{r}\left[f_{r}+\frac{2 K_{r}}{c}\left(r_{0}-r_{r e f}\right)\right]\right\} \exp \left(-j \frac{4 \pi}{c} f_{0} r_{0}+j \pi K_{a} \eta_{0}^{2}\right) \exp \left(j 2 \pi K_{a} \eta_{l} \eta_{0}\right)
\end{aligned}
$$

where $r_{\Delta}=r\left(\eta_{l}, r_{0}\right)-r_{\text {ref }}, t_{n}$ and $\eta_{l}$ denote the number of samples in range and azimuth dimension, respectively. Specifically, the structural composition of vectors $\mathbf{s}_{r}, \mathbf{s}_{r e f}, \mathbf{s}_{i f}$ and $\mathbf{S}$ can be expressed 


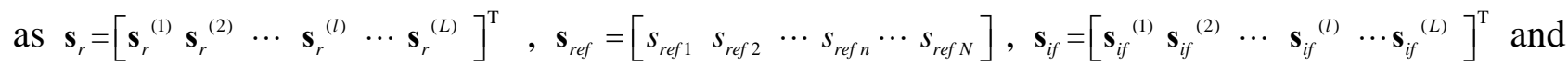
$\mathbf{S}=\left[\begin{array}{llllll}\mathbf{S}^{(1)} & \mathbf{S}^{(2)} & \cdots & \mathbf{S}^{(l)} & \cdots & \mathbf{S}^{(L)}\end{array}\right]^{\mathrm{T}}$, where $\mathbf{s}_{r}{ }^{(l)}=\left[\begin{array}{llllll}s_{r l, 1} & s_{r l, 2} & \cdots & s_{r l, n} & \cdots & s_{r l, N}\end{array}\right]$ and $\mathbf{s}_{i f}{ }^{(l)}=\left[\begin{array}{llllll}s_{i f l, 1} & s_{i f l, 2} & \cdots & s_{i f l, n} & \cdots & s_{i f l, N}\end{array}\right]$. Construct a diagonal matrix $\mathbf{D} \in \mathbb{C}^{N \times N}$ whose diagonal elements are the components of $\mathbf{s}_{\text {ref }}$

$$
\mathbf{D}(q, p)=\left\{\begin{array}{ll}
\mathbf{s}_{r e f}(q) & q=p \\
0 & q \neq p
\end{array}, 1 \leq q, p \leq N\right.
$$

Assume $\mathbf{F}_{r} \in \mathbb{C}^{N \times N}$ and $\mathbf{F}_{a} \in \mathbb{C}^{L \times L}$ are Fourier transform matrixes

$$
\begin{aligned}
& \mathbf{F}_{r}=\exp \left(-j 2 \pi \frac{q}{N} p\right), 1 \leq q, p \leq N \\
& \mathbf{F}_{a}=\exp \left(-j 2 \pi \frac{q}{L} p\right), 1 \leq q, p \leq L
\end{aligned}
$$

From Eq.(3) we can get $\mathbf{s}_{i f}{ }^{(l)}=\mathbf{D}^{\mathrm{H}} \mathbf{s}_{r}{ }^{(l)}$, from Eq.(4) we can get $\mathbf{s}_{i f}{ }^{(l)}=\mathbf{F}_{r} \mathbf{h}^{(l)}$ and from Eq.(6) we can get $\mathbf{S}=\mathbf{F}_{a} \boldsymbol{\sigma}$, where the vector $\mathbf{h}^{(l)}$ represents the targets range profile at the lth azimuth cell and the vector $\boldsymbol{\sigma}$ represents the targets reflectivity, consequently these can be represented as

$$
\begin{aligned}
& \mathbf{s}_{r}^{(l)}=\mathbf{D} \mathbf{F}_{r} \mathbf{h}^{(l)}=\boldsymbol{\Psi}_{r} \mathbf{h}^{(l)}, \quad \boldsymbol{\Psi}_{r}=\mathbf{D} \mathbf{F}_{r} \\
& \mathbf{S}=\mathbf{F}_{a} \boldsymbol{\sigma}=\boldsymbol{\Psi}_{a} \boldsymbol{\sigma}, \boldsymbol{\Psi}_{a}=\mathbf{F}_{a}
\end{aligned}
$$

where $\boldsymbol{\Psi}_{r}$ represents the range sparse matrix and $\boldsymbol{\Psi}_{a}$ represents the azimuth sparse matrix.

Then, assume $\boldsymbol{\Phi}_{r}^{(l)} \in \mathbb{C}^{M \times N}(M<<N)$ and $\boldsymbol{\Phi}_{a} \in \mathbb{C}^{P \times L}(P<<L)$ are the random downsampling measurement matrixes in range and azimuth dimension respectively, the randomly sampling measurements are

$$
\begin{aligned}
& \mathbf{y}_{r}^{(l)}[m]=\boldsymbol{\Phi}_{r}^{(l)} \mathbf{s}_{r}^{(l)}=\boldsymbol{\Phi}_{r}^{(l)} \boldsymbol{\Psi}_{r} \mathbf{h}^{(l)} \\
& \mathbf{y}_{a}[p]=\boldsymbol{\Phi}_{a} \mathbf{S}=\boldsymbol{\Phi}_{a} \boldsymbol{\Psi}_{a} \boldsymbol{\sigma}
\end{aligned}
$$

At the end, the OMP algorithm is used to reconstruct the targets range profiles $\mathbf{h}=\left[\mathbf{h}^{(1)}, \mathbf{h}^{(2)} \cdots \mathbf{h}^{(l)} \cdots \mathbf{h}^{(L)}\right]$ and targets reflectivity $\boldsymbol{\sigma}$.

The steps of SAR imaging method based on CS are as follows.

Step1 Construct the range measurement matrixes $\boldsymbol{\Phi}_{r}^{(1)}, \boldsymbol{\Phi}_{r}^{(2)}, \ldots \boldsymbol{\Phi}_{r}^{(l)} \ldots, \boldsymbol{\Phi}_{r}^{(L)}$, then we get the measurements $\mathbf{y}_{r}^{(l)}$ at the lth azimuth cell according to Eq.(14).

Step2 Construct range sparse matrix $\boldsymbol{\Psi}_{r}$ from Eq.(13) and reconstruct range profile $\mathbf{h}^{(l)}$ by the OMP algorithm.

Step3 Repeat Step1 and Step2 to get a series of range profiles $\mathbf{h}$.

Step4 Based on range compression, conduct the phase compensation and stretch processing in azimuth dimension.

Step5 Construct azimuth sparse matrix $\boldsymbol{\Psi}_{a}$ from Eq.(13) and azimuth measurement matrix $\boldsymbol{\Phi}_{a}$. Then reconstruct the targets reflectivity $\boldsymbol{\sigma}$ from $\mathbf{y}_{a}$ by the OMP algorithm.

\section{Simulations}

To verify the performance of the proposed method, simulations are presented in this section. The system transmits LFM signal. Suppose the carrier frequency is $f_{0}=1 \mathrm{GHz}$, the range pulse duration is $T_{r}=5 u s$, the bandwidth is $B=100 \mathrm{MHz}$, the pulse repeat frequency (PRF) is $400 \mathrm{~Hz}$, the velocity is $v=100 \mathrm{~m} / \mathrm{s}$, the platform height is $H=1000 \mathrm{~m}$ and 4 point targets are set in the imaging area.

Fig. 2 show the range profile and targets images obtained by conventional SP method. We randomly sample with $50 \%$ of the signal data in range and azimuth dimension respectively. The reconstructed range profile and targets images by the proposed CS method are shown in Fig. 3. Compared Fig. 3(a) with Fig. 2(a), it can be found that the CS method has much lower sidelobe than the conventional SP method. It can be also observed form Fig. 2(b) and Fig. 3(b) that the imaging quality based on CS method is superior to SP method with lower samples at the same time. 
We add the Gaussian noise to the echo signal and the results are shown in Fig. 4, the method based on CS is able to reconstruct the targets images with SNR from $20 \mathrm{~dB}$ to 0dB. The targets images can still be reconstructed perfectly when we further down sample the echo signal to $10 \%$ data in range and azimuth dimension respectively. The results are shown in Fig. 5.

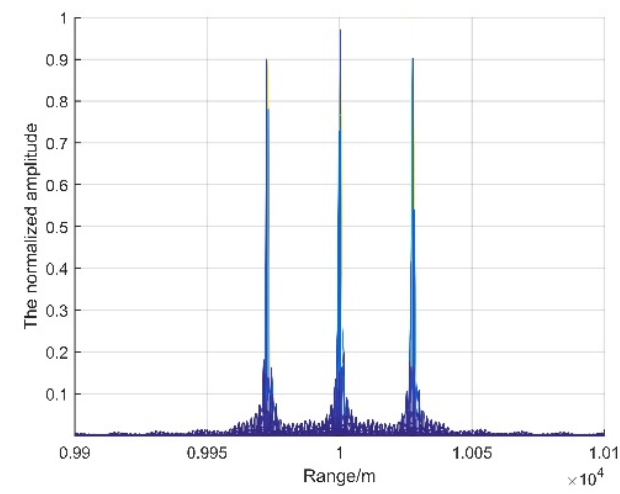

(a) The obtained range profile

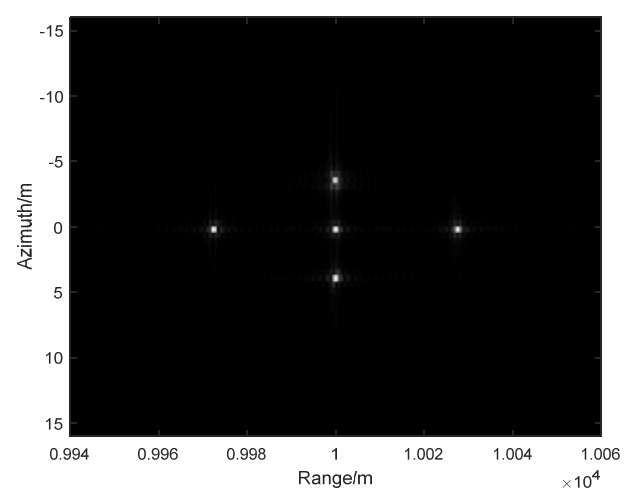

(b) The targets images

Fig. 2 SAR imaging results based on conventional SP method

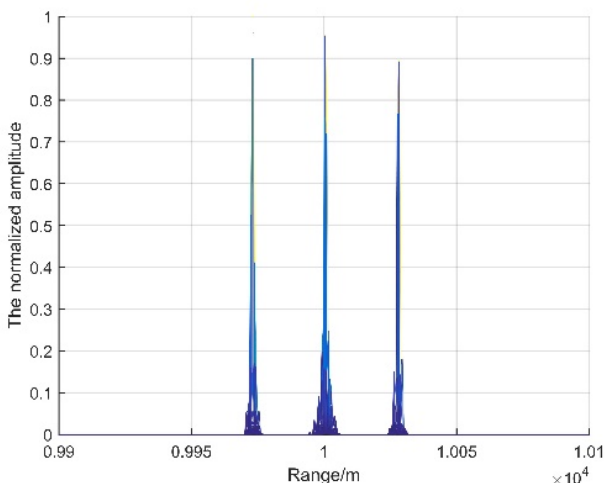

(a) The obtained range profile

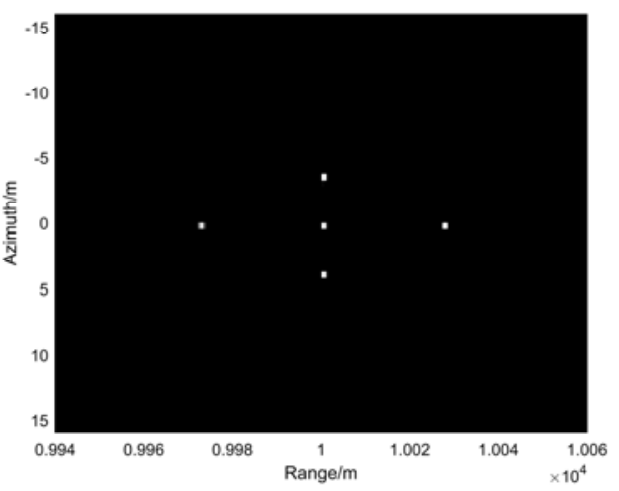

(b) The targets images

Fig. 3 SAR imaging results based on CS method with 50\% samples in both range and azimuth
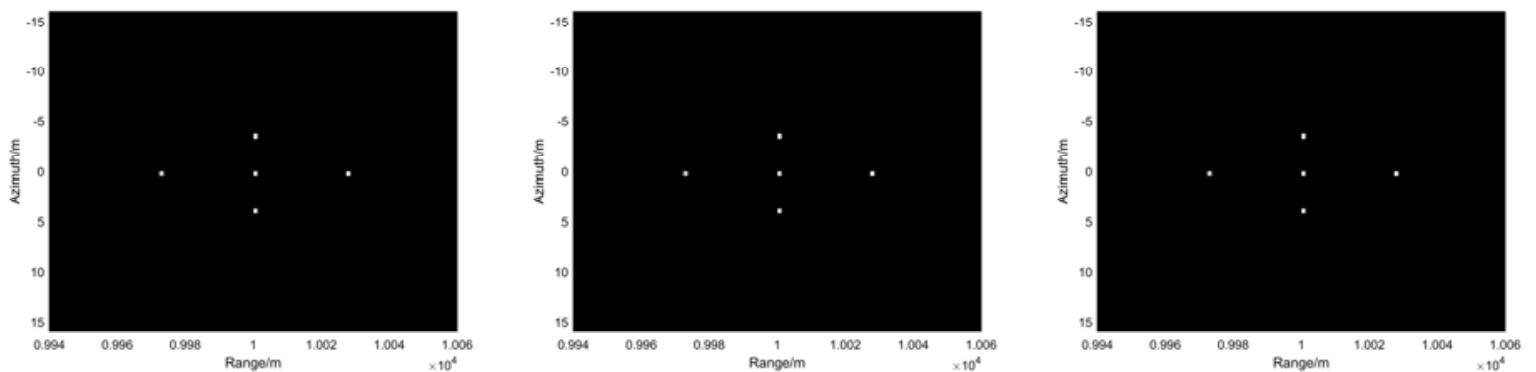

Fig. 4 reconstruction results with $50 \%$ samples in both range and azimuth at SNRs are $20 \mathrm{~dB}, 10 \mathrm{~dB}$ and $0 \mathrm{~dB}$, respectively
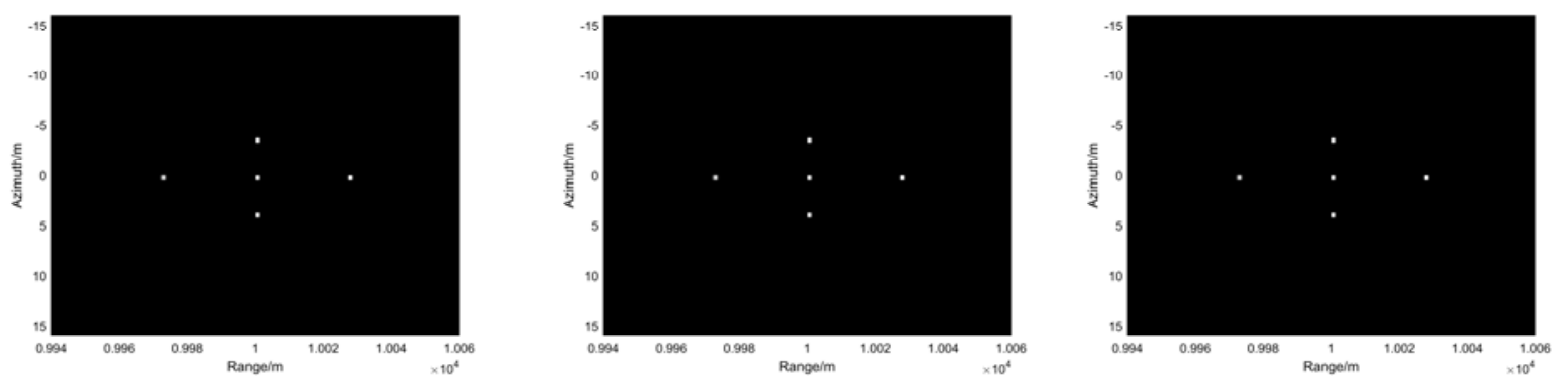

Fig. 5 reconstruction results with $10 \%$ samples in both range and azimuth at SNRs are $20 \mathrm{~dB}, 10 \mathrm{~dB}$ and $0 \mathrm{~dB}$, respectively 


\section{Summary}

This paper proposes a novel SAR imaging method based on CS for wideband LFM signal. Essentially, it has proposed an alternative to the conventional stretch processing for SAR raw data. In the method, CS theory is applied into two 1-D processing operations: range and azimuth. A range sparse matrix based on stretch processing and Fourier transform is constructed and then applied to obtain the range profile information. Based on the range compression, the azimuth compression processing is conducted. The method replaces the coherent mixing processing with the sparse matrix construction, eliminating the mixer in radar system, consequently simplifies the complexity of hardware in radar system. Compared with conventional SP method, the proposed CS method significantly suppresses sidelobe and effectively reduces the number of samples that required in the Nyquist sampling theorem. Besides, the method is robust in the case of strong noise.

This work is supported by National Nature Science Foundation of China 61571229 and National Nature Science Foundation of China 61271331.

\section{References}

[1]. Donoho D L. Compressed sensing [J]. IEEE Transactions on information theory. 2006, 52(4): 1289-1306.

[2]. Candès E J, Romberg J, Tao T. Robust uncertainty principles: Exact signal reconstruction from highly incomplete frequency information [J]. IEEE Transactions on information theory. 2006, 52(2): 489-509.

[3]. Potter L C, Ertin E, Parker J T, et al. Sparsity and compressed sensing in radar imaging [J]. Proceedings of the IEEE. 2010, 98(6): 1006-1020.

[4]. Eldar, Yonina C, Gitta Kutyniok, et al. Compressed sensing: theory and applications [M]. Cambridge University Press, 2012.

[5]. Liu J, Xu S, Gao X, et al. Novel imaging methods of stepped frequency radar based on compressed sensing [J]. Journal of Systems Engineering and Electronics. 2012, 23(1): 47-56.

[6]. Baraniuk R and Steeghs P. Compressed radar imaging[C]. IEEE Radar Conference. Boston, MA, USA, Apr.17-20, 2007, 128-133.

[7]. Alonso M T, López-Dekker P, Mallorquí J J. A novel strategy for radar imaging based on compressed sensing [J]. IEEE Transactions on Geoscience and Remote Sensing. 2010, 48(12): 4285-4295.

[8]. Yang J, Thompson J, Huang X, et al. Segmented reconstruction for compressed sensing SAR imaging [J]. IEEE transactions on geoscience and remote sensing. 2013, 51(7): 4214-4225.

[9]. Dong X, Zhang Y. A novel compressed sensing algorithm for SAR imaging [J]. IEEE Journal of selected topics in applied earth observations and remote sensing. 2014, 7(2): 708-720.

[10]. Bu H, Tao R, Bai X, et al. A novel SAR imaging algorithm based on compressed sensing [J]. IEEE Geoscience and Remote Sensing Letters. 2015, 12(5): 1003-1007.

[11]. Fang J, Xu Z, Zhang B, et al. Fast compressed sensing SAR imaging based on approximated observation [J]. IEEE Journal of Selected Topics in Applied Earth Observations and Remote Sensing. 2014, 7(1): 352-363.

[12]. Gao L, Su S Y, Chen Z P. Orthogonal sparse representation for chirp echoes in broadband radar and its application to compressed sensing [J]. Journal of Electronics and Information Technology. 2011, 33(11): 2720-2726. 